\title{
Walking in simulated reduced gravity: mechanical energy fluctuations and exchange
}

\author{
TIMOTHY M. GRIFFIN, NEIL A. TOLANI, AND RODGER KRAM \\ Department of Integrative Biology, University of California, Berkeley, California 94720-3140
}

\begin{abstract}
Griffin, Timothy M., Neil A. Tolani, and Rodger Kram. Walking in simulated reduced gravity: mechanical energy fluctuations and exchange. J . Appl. Physiol. 86(1): 383-390, 1999.-Walking humans conserve mechanical and, presumably, metabolic energy with an inverted pendulum-like exchange of gravitational potential energy and horizontal kinetic energy. Walking in simulated reduced gravity involves a relatively high metabolic cost, suggesting that the invertedpendulum mechanism is disrupted because of a mismatch of potential and kinetic energy. We tested this hypothesis by measuring the fluctuations and exchange of mechanical energy of the center of mass at different combinations of vel ocity and simulated reduced gravity. Subjects walked with smaller fluctuations in horizontal velocity in lower gravity, such that the ratio of horizontal kinetic to gravitational potential energy fluctuations remained constant over a fourfold change in gravity. The amount of exchange, or percent recovery, at $1.00 \mathrm{~m} / \mathrm{s}$ was not significantly different at 1.00 , 0.75 , and $0.50 \mathrm{G}$ (average $64.4 \%$ ), although it decreased to $48 \%$ at $0.25 \mathrm{G}$. As a result, the amount of work performed on the center of mass does not explain the relatively high metabolic cost of walking in simulated reduced gravity.
\end{abstract}

biomechanics; locomotion; human; mechanical work; energetics

"GRAVITY NOT ONLY CONTROLS the actions but also the forms of all save the least of organisms" (32). Despite our intuitive appreciation for the influence of gravity, we do not understand how gravity interacts with other forces, such as inertia, to affect many biological and physical processes. Such is the case for walking. The goal of this study was to use simulated reduced gravity to gain a better understanding of how gravity affects the mechanics and energetics of walking.

Walking is characterized by a pendulum-like exchange of the gravitational potential energy $\left(E_{p}\right)$ and horizontal kinetic energy $\left(E_{k h}\right)$ of the body's center of mass. This mechanism conserves mechanical energy and presumably metabolic energy. Cavagna et al. (11) found that the vertical position and forward velocity of the body's center of mass fluctuate "out of phase" during each step cycle. During the middle of the stance phase, when the body is over the relatively stiff support leg, the vertical position of the body is highest, whereas the forward velocity is slowest. As a result, the body's $E_{p}$ reaches a maximum at midstance, whereas the $E_{k h}$ reaches a minimum. During the second half of the stance phase the center of mass falls and increases

\footnotetext{
The costs of publication of this article were defrayed in part by the payment of page charges. The article must therefore be hereby marked "advertisement" in accordance with 18 U.S.C. Section 1734 solely to indicate this fact.
}

velocity. Consequently, at the end of the stance phase the $E_{p}$ is low and the $E_{k h}$ is high.

During each step cycle the kinetic and potential energy are exchanged such that the total external work required to lift and accelerate the body's center of mass is less than the sum of the positive increments in potential and kinetic energy (12). This exchange is anal ogous to the exchange of energy in a simple pendulum. As a result, many investigators have modeled walking as an inverted pendulum, in which body mass is ideal ized to a point mass on massless rigid legs $(2,12$, 29). The amount of exchange, often described as "percent recovery," is greatest at moderate walking speeds ( $\sim 1.5 \mathrm{~m} / \mathrm{s}$ for adults), because the energy fluctuations are nearly equal in magnitude and are $180^{\circ}$ out of phase. However, unlike an ideal pendulum, which has $100 \%$ recovery, humans normally recover only $\sim 65 \%$ of the total mechanical energy $\left(E_{\text {tot }}\right)$, because the energies do not fluctuate at opposite equal rates (i.e., the shapes of the energy fluctuation-time curves are not mirror images). As a result, mechanical energy must be added to the system by the leg muscles during each step. Studies of mechanical energy fluctuations in a wide variety and size range of terrestrial animals, from lizards to sheep, have shown that this basic energysaving mechanism is characteristic of walking gaits (5, $10,15)$

Before the Apollo Missions to the Moon, human locomotion in reduced gravity was the subject of much interest and research $(18,21)$. Margaria and Cavagna $(24,25)$ were among the first to speculate about the effect of reduced gravity on the mechanical energy fluctuations in walking humans. They theorized that at a constant walking speed the inertial forces would not change as gravity was reduced, and thus they predicted that reduced gravity would change the ratio of potential to kinetic energy fluctuations. The premise for their prediction was that less mechanical work would be performed against gravity per step, and, subsequently, less potential energy would be available to convert to kinetic energy and accelerate the body. A natural extension of their prediction was that, as gravity was reduced, the recovery of mechanical energy would decrease, and the amount of external work performed on the body's center of mass would increase. This reasoning assumed that at a given speed the vertical displacements and forward velocity fluctuations of the center of mass remained the same as gravity was reduced. Although many other aspects of locomotion in reduced gravity have been examined $(13,30)$, the theoretical speculation of Margaria and Cavagna has not been empirically tested in the ensuing 35 years.

However, one study of the metabolic cost of walking in simulated reduced gravity appears to support the 
speculation of Margaria and Cavagna (24, 25). Farley and McMahon (16) found that when gravity was reduced by $50 \%$ the metabolic cost of walking decreased by only $25 \%$. Considering that the metabolic cost of running in simulated reduced gravity decreased in direct proportion to gravity, reducing gravity had a comparatively small effect on the metabolic cost of walking. Farley and McMahon proposed that their results for walking reflected an increase in the amount of work performed by the muscles because of an ineffective exchange of mechanical energy and a simultaneous decrease in the energetic cost of generating muscular force to support the reduced body weight. Previously, it had been assumed that the mechanical work alone could account for the metabolic cost of walking, whereas the metabolic cost of generating muscular force isometrically to maintain joint stiffness and stability was negligible (12). However, if work increased in reduced gravity, the results from the study of Farley and McMahon suggest that the cost of generating muscular force to support body weight is substantial. Consequently, understanding how reduced gravity affects the exchange of mechanical energy may provide important insight into the determinants of the metabolic cost of walking in normal gravity.

The goal of this study was to determine the separate and combined effects of speed and simulated reduced gravity on the mechanical energy fluctuations and exchange in walking. On the basis of the predicted mismatch of potential and kinetic energies in low gravity, we hypothesized that 1) at a moderate speed the recovery of mechanical energy would decrease as gravity is reduced and 2) maximum recovery would occur at progressively slower speeds as gravity is reduced. In addition to recovery, we quantified the amount of external mechanical work performed during walking across a range of speeds and gravity levels.

\section{METHODS}

General procedures. Six subjects [3 men and 3 women; average age $=24.2 \pm 6.0$ (SD) yr, average mass $=64.9 \pm 14.5$ $\mathrm{kg}$, average leg length $=90.5 \pm 5.7 \mathrm{~cm}$ ] provided informed consent to take part in the study. All were experienced treadmill walkers and were familiarized with our reducedgravity simulator. The subjects walked on a force treadmill (FTM; see below) in normal gravity, i.e., $1.00 \mathrm{G}$ (where G is "times normal gravity"), and in conditions simulating 0.75 , 0.50 , and $0.25 \mathrm{G}$. At each level of gravity the subjects were studied at speeds of $0.50,0.75,1.00,1.25,1.50,1.75$, and 2.00 $\mathrm{m} / \mathrm{s}$. After a warm-up, a $\geq 1$-min habituation period was allowed for each condition before the actual data collection was begun. Donelan and Kram (14) demonstrated that walking kinematics rapidly adapt to simulated reduced gravity. They found that duty factor (the fraction of a stride time that a foot is in contact with the ground) and stride length changed by $<1 \%$ between the 2 nd and 6 th min in normal gravity and by 1 and $3 \%$, respectively, in $0.25 \mathrm{G}$. They concluded that 1 min under each condition was sufficient to familiarize subjects with reduced-gravity treadmill walking. In our study, each subject walked at a total of 28 different combinations of speed and gravity. Data were collected and analyzed for five strides for each subject in every condition.
Reduced-gravity simulator. We simulated reduced gravity with an apparatus that applied a nearly constant upward force to the body near the overall body center of mass (Fig. 1). This apparatus was similar to that described by Kram et al. (19). The force was applied via a modified rock-climbing harness strapped about the subject's waist and pelvis. The harness was held in suspension by an aluminum $\mathrm{H}$-shaped bar above the subject's head that stabilized the vertical straps of the harness away from the subject's chest and arms.

Attached to the $\mathrm{H}$-shaped bar was a cable that was in line with a dual winch-and-pulley system (Fig. 1). The system consisted of a low-friction rolling trolley, four sets of compliant rubber tubing in series with the cable, and two winches, one at each end of the cable line. The rolling-trolley mechanism, inspired by Letko and Spady (22), ensured that only a vertical force was applied to the subject regardless of the subject's position along the length of the treadmill. The rubber tubing el ements acted as springs, which were stretched to simulate the desired levels of gravity with fluctuations of less than $\pm 0.06 \mathrm{G}$. Additional parallel springs were added only when the force applied by the original springs was insufficient.

Our reduced-gravity simulator did not exactly provide the effect that true reduced gravity would have on the swinging of the limbs. Thus our results could vary from those obtained in true reduced gravity. However, Donelan and Kram (14), who used essentially the same simulator, found walking kinematics similar to those in studies involving moreintricate reducedgravity simulations that do unweight the limbs $(6,13)$. Precursors to our simulator have been used successfully in many prior experiments $(14,16,17,19$; see Refs. 13 and 14 for a more complete discussion of methods for simulating reduced gravity).

FTM. The FTM used in this study has been described in detail recently by Kram et al. (20). The FTM consisted of a custom-made treadmill mounted onto a commercial straingauged multicomponent force platform (model ZBP-7124-64000, AMTI , Watertown, MA). The treadmill was lightweight,

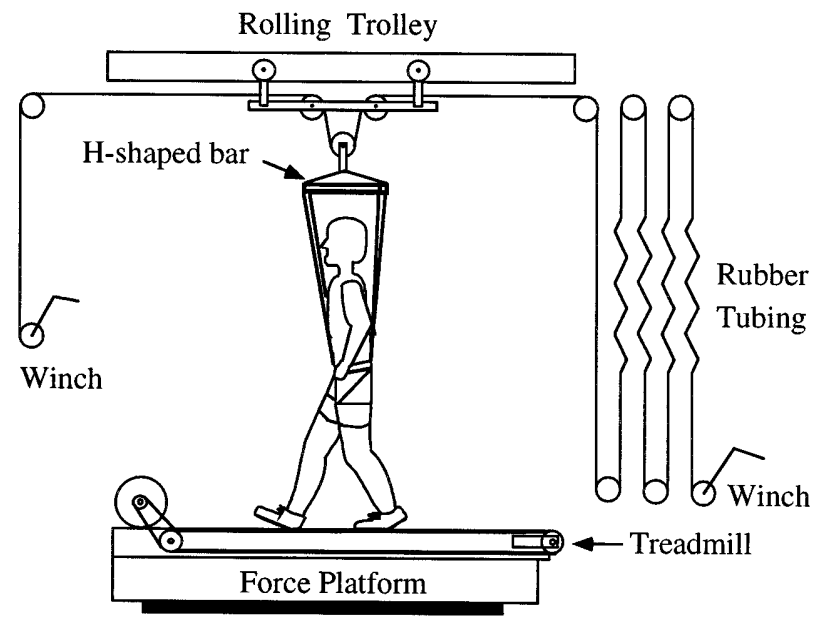

Fig. 1. Reduced-gravity simulator and force treadmill. Reducedgravity simulator applied a nearly constant upward force near the body's center of mass via a pelvic harness. Rolling-trolley mechanism ensured that only a vertical force was applied to subject. Magnitude of force was increased by lengthening rubber spring elements with winches. To maximize spring stretch and attenuate force fluctuations, additional rubber springs were added in parallel only when force of original springs was insufficient. Level of simulated reduced gravity was determined by measuring body weight with force treadmill, which also recorded vertical and horizontal ground reaction forces. Details of force treadmill are provided in Ref. 20. 
mechanically stiff, and supported along its length by the force platform. The FTM measured the vertical and horizontal (anterior-posterior) ground reaction forces $\left(F_{z}\right.$ and $F_{y}$, respectively).

Analysis of human walking ground reaction forces indicates that $99 \%$ of the integrated power content is at frequencies $<10 \mathrm{~Hz}$ for the $\mathrm{F}_{z}$ and $\mathrm{F}_{\mathrm{y}}$ signals (20). Because the natural frequencies of vibration of the FTM ranged from 180 to $80 \mathrm{~Hz}$ $\left(F_{z}\right.$ and $F_{y}$, respectively), we are confident that the FTM accurately recorded the ground reaction forces for human walking. A comparison of ground reaction forces obtained from a force platform runway with filtered FTM data verified that the FTM accurately recorded the ground reaction forces (20).

For each condition we collected force data at $1 \mathrm{kHz}$ for a 10 -s period. Subsequently, the data were filtered using a fourth-order low-pass Butterworth nonrecursive filter passed in both directions to effect zero phase shift and a 3-dB cutoff of $20 \mathrm{~Hz}$. This filtering process only slightly affected data with frequencies $<10 \mathrm{~Hz}$. After the raw force data were filtered, the mechanical energy fluctuations of the center of mass were calculated.

Mechanical energy cal culations. We cal culated the mechanical energy fluctuations of the center of mass by using the integration process previously described in detail by Cavagna (7). Kinetic energy and $E_{p}$ fluctuations were calculated per stride by determining the velocity and position changes, respectively, of the center of mass. A Labview 4.01 (National Instruments) integration program was used to determine the instantaneous velocity and position of the center of mass during the stride. Integration constants were chosen according to Cavagna (7); however, with our FTM, the average forward velocity was equal to the treadmill belt speed. $E_{\text {tot }}$ fluctuations were cal culated as the instantaneous sum of $E_{k h}$, vertical kinetic energy $\left(E_{k v}\right)$, and $E_{p}$ of the center of mass. Overall, $E_{k v}$ had a negligible effect on the fluctuations of $E_{\text {tot }}$, because it was very small compared with the fluctuations in $E_{p}$ and $E_{k h}$ (12). We quantified the fluctuations in $E_{t o t}, E_{k h}$, $E_{k v}$, and $E_{p}$ by summing the positive increments per stride.

The work performed per stride was cal culated as the sum of the positive increments in mechanical energy. The vertical work per stride $\left(\mathrm{W}_{\mathrm{v}}\right)$ was equal to the positive increments of the fluctuations in vertical energy. Vertical energy was calculated as the sum of $E_{p}$ and $E_{k v}$. Because $E_{k v}$ was zero when $E_{p}$ was at a maximum or minimum during the stride, $E_{k v}$ did not contribute to $W_{v}$. The forward work $\left(W_{f}\right)$ was equal to the positive increments of the $E_{k h}$ fluctuations. The positive increments in $E_{\text {tot }}$ fluctuations were equal to the total external work performed per stride $\left(W_{\text {ext }}\right)$. In walking, $W_{\text {ext }}$ is always less than the sum of $W_{v}$ and $W_{f}$, since mechanical energy is recovered within the stride.
Percent recovery of mechanical energy of thecenter of mass was cal culated according to Eq. 1 given by Cavagna et al . (12)

$$
\% \text { recovery }=\frac{W_{v}+W_{f}-W_{\text {ext }}}{W_{v}+W_{f}} \cdot 100
$$

The magnitude of $W_{\text {ext, }}$ and thus the percent recovery of mechanical energy, depends on the relative magnitudes of $W_{f}$ and $W_{v}$, the phase difference $(\alpha)$ of the fluctuations in $E_{k h}$ and $\left(E_{p}+E_{k v}\right)$, and the rates of fluctuation (shapes of the curves) of $E_{k h}$ and $\left(E_{p}+E_{k v}\right)$. We calculated the rel ative magnitude of $W_{f}$ and $W_{v}$ as the ratio $\left(W_{f} / W_{v}\right)$ for each stride. The $\alpha$ values between the fluctuations in $E_{k h}$ and $\left(E_{p}+E_{k v}\right)$ were calculated according to $\mathrm{Eq}$. 2 from Cavagna et al. (9)

$$
\alpha=360^{\circ} \cdot \frac{\Delta t}{T}
$$

where $\Delta t$ is the difference between the time at which $E_{k h}$ was maximum and $\left(E_{p}+E_{k v}\right)$ was minimum and $T$ is the step period (i.e., the period of repeating changes in forward and vertical velocity of the center of mass). As defined here, each stride consisted of two step periods. Given this definition of $\alpha$, if $E_{k h}$ and $\left(E_{p}+E_{k v}\right)$ fluctuated $180^{\circ}$ out of phase, $\alpha$ would be equal to $0^{\circ}$. We did not quantify or compare the rates of fluctuations (i.e., shapes of the curves) of $E_{k h}$ and $\left(E_{p}+E_{k v}\right)$, because an appropriate method has yet to be devel oped.

Several studies have reported that the preferred walk-run transition speed decreases at lower levels of gravity $(19,34)$. Thus we restricted our data analysis to walking gaits, defined as a gait in which the center of mass reaches its highest point near the middle of the stance phase (26). Midstance was determined by analyzing the $F_{y}$ traces. We defined midstance as the instant during single limb support when $F_{y}$ crossed zero. A trial was accepted if the vertical position of the center of mass at midstance was closer to its highest point than its lowest point during the step. Trials that did not meet this criterion were not used for analysis.

Statistical analysis. In all instances, a repeated-measures ANOVA was used to determine statistical significance with $\mathrm{P}<0.05$. Our statistical analyses were carried out with the software package J MP IN (SAS I nstitute).

\section{RESULTS}

Fluctuations and exchange at $1.00 \mathrm{~m} / \mathrm{s}$. We began by examining the mechanical energy fluctuations of the center of mass at $1.00 \mathrm{~m} / \mathrm{s}$ in normal gravity (1.00 G) and three different levels of simulated reduced gravity $(0.75,0.50$, and $0.25 \mathrm{G})$. A summary of these results is given in Table 1 . We chose to analyze $1.00 \mathrm{~m} / \mathrm{s}$ so we

\begin{tabular}{|c|c|c|c|c|c|}
\hline & $1.00 \mathrm{G}$ & $0.75 \mathrm{G}$ & $0.50 \mathrm{G}$ & $0.25 \mathrm{G}$ & $P$ \\
\hline Recovery, \% & $64.0 \pm 2.9$ & $65.3 \pm 1.5$ & $64.0 \pm 3.6$ & $48.7 \pm 5.3^{*}$ & $<0.05$ \\
\hline $\mathrm{W}_{\mathrm{f}} / \mathrm{W}_{\mathrm{v}}$ & $0.92 \pm 0.09$ & $0.89 \pm 0.07$ & $0.93 \pm 0.05$ & $0.97 \pm 0.07$ & 0.70 \\
\hline$\alpha$, degrees & $22.0 \pm 5.2$ & $-4.1 \pm 9.2$ & $-27.3 \pm 7.5$ & $-34.1 \pm 6.5$ & $<0.01$ \\
\hline $\mathrm{W}_{\mathrm{f}}, \mathrm{J} \cdot \mathrm{kg}^{-1} \cdot \mathrm{stride}^{-1}$ & $0.40 \pm 0.02$ & $0.34 \pm 0.01$ & $0.22 \pm 0.02$ & $0.12 \pm 0.01$ & $<0.01$ \\
\hline $\mathrm{W}_{\mathrm{v}}, \mathrm{J} \cdot \mathrm{kg}^{-1} \cdot \mathrm{stride}^{-1}$ & $0.47 \pm 0.06$ & $0.40 \pm 0.04$ & $0.25 \pm 0.02$ & $0.13 \pm 0.02$ & $<0.01$ \\
\hline $\mathrm{W}_{\text {ext }}, \mathrm{J} \cdot \mathrm{kg}^{-1} \cdot \mathrm{stride}^{-1}$ & $0.31 \pm 0.03$ & $0.26 \pm 0.02$ & $0.17 \pm 0.02$ & $0.13 \pm 0.02$ & $<0.01$ \\
\hline $\mathrm{S}_{\mathrm{v}}, \mathrm{cm} /$ stride & $4.8 \pm 0.6$ & $5.4 \pm 0.5$ & $5.1 \pm 0.5$ & $5.2 \pm 0.7$ & 0.74 \\
\hline
\end{tabular}

Table 1. Data for walking at $1.00 \mathrm{~m} / \mathrm{s}$ at a range of gravity levels

Values are means $\pm S E ; n=6$ subjects. $S_{v}$, vertical displacement of center of mass. $W_{v}$, vertical work per stride; $W_{f}$, forward work per stride; $W_{\text {ext }}$, total external work per stride; $\alpha$, phase difference. P values were determined by ANOVA. *Tukey's honestly significant difference test revealed that $1.00-, 0.75-$, and $0.50-G$ recovery values were not significantly different, but $0.25-G$ value was significantly different $(P<0.05)$. 
could compare our results with the metabolic data of Farley and McMahon (16). The fluctuations in $E_{p}, E_{k n}$, and $E_{\text {tot }}$ over one stride are shown in Fig. 2 for a typical subject walking at $1.00 \mathrm{~m} / \mathrm{s}$. As gravity was reduced, the fluctuations in $E_{p}$ and $E_{k h}$ decreased nearly in proportion with gravity (Table 1, Fig. 2). As a result, $\mathrm{W}_{\mathrm{f}} \mathrm{W}_{\mathrm{v}}$ remained unchanged over a fourfold change in gravity during walking at $1.00 \mathrm{~m} / \mathrm{s}$ (Table 1). In contrast, reducing gravity changed the magnitude and direction of $\alpha$ between the fluctuations in $\mathrm{E}_{\mathrm{kh}}$ and $\left(\mathrm{E}_{\mathrm{p}}+\right.$ $E_{k v}$ ) (Table 1 ). $W_{\text {ext }}$ was also affected by gravity. The fluctuations in $\mathrm{E}_{\text {tot }}$ decreased as gravity was reduced (Fig. 2). The mass-specific external work per stride decreased by $58 \%$ when gravity was reduced by $75 \%$ (Table 1). Percent recovery, on the other hand, was not
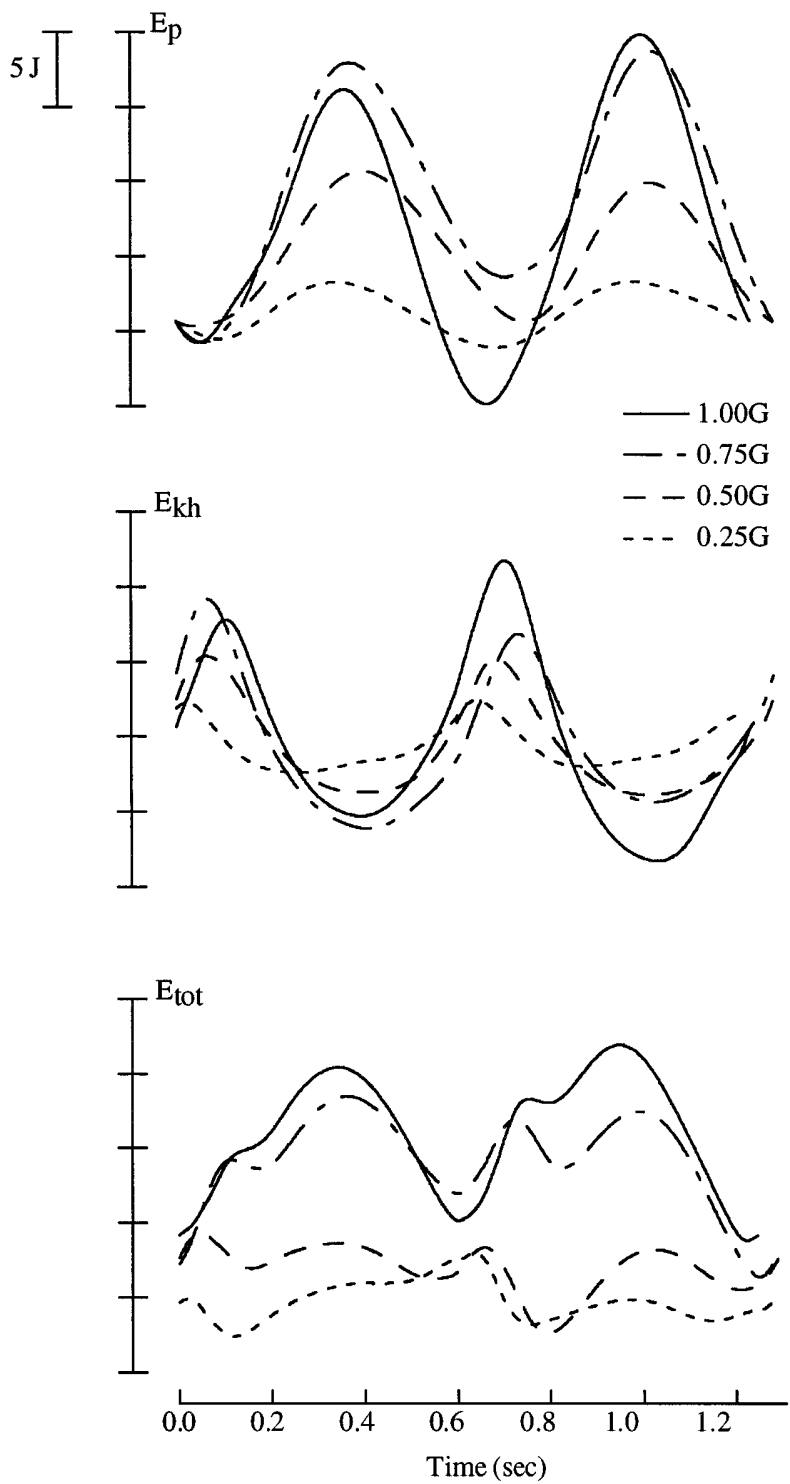

Fig. 2. Fluctuations in mechanical energy of center of mass during a stride for a typical subject walking at $1.00 \mathrm{~m} / \mathrm{s}$. Fluctuations in gravitational potential energy $\left(E_{p}\right)$ and horizontal kinetic energy $\left(E_{k h}\right)$ of center of mass were generally out of phase, and so $E_{p}+E_{k h}$ (total mechanical energy, $E_{\text {tot }}$ ) exhibited smaller fluctuations. Positive increments in $E_{\text {tot }}$, i.e., work performed, decreased as gravity was reduced.

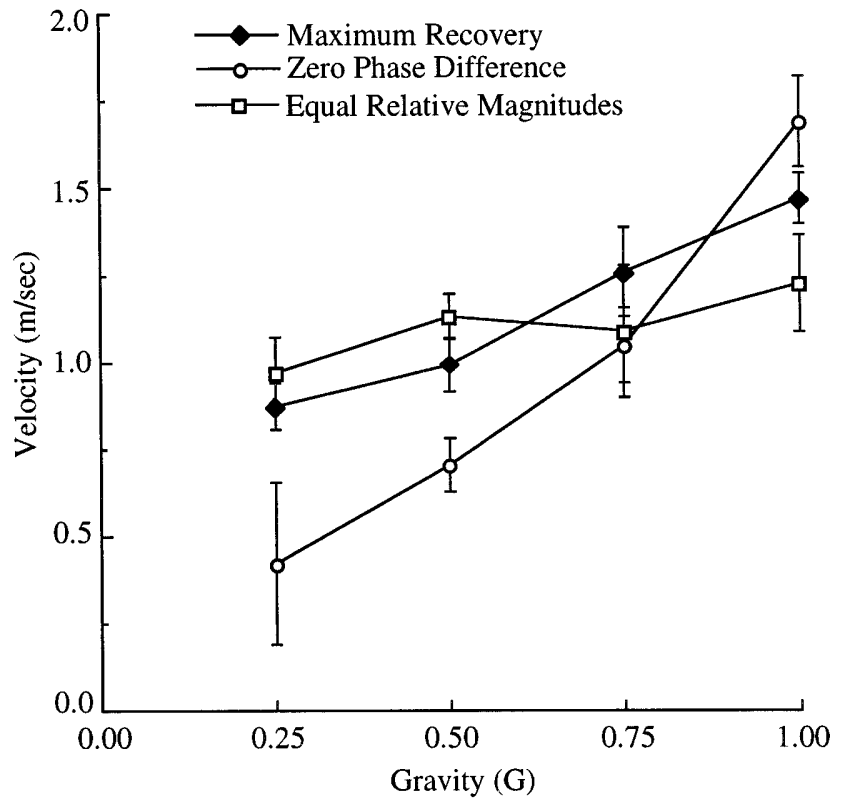

Fig. 3. Velocity with greatest recovery was slower in reduced gravity. Potential and kinetic energies fluctuated $180^{\circ}$ out of phase [phase difference $(\alpha)=0^{\circ}$ ] at progressively slower velocities as gravity was reduced. However, velocity where the relative magnitude of forward to vertical work $\left(\mathrm{W}_{\mathrm{f}} / \mathrm{W}_{\mathrm{v}}\right)=1.0$ was only slightly affected by gravity. Velocities were calculated for each subject and condition by applying a linear or 2nd-order polynomial curve fit to data and solving for velocity according to given criteria (e.g., $\alpha=0^{\circ}$ ). Values are means \pm SE for 6 subjects.

significantly affected by a $50 \%$ reduction in gravity. However, recovery did decrease slightly at $0.25 \mathrm{G}$ (Table 1). The near independence of recovery and gravity at $1.00 \mathrm{~m} / \mathrm{s}$ appears to be due to the constant $\mathrm{W}_{\mathrm{f}} / \mathrm{W}_{\mathrm{v}}$ and to the decreased fluctuations in $\mathrm{E}_{\text {tot }}$ at lower levels of gravity.

Fluctuations and exchange as a function of speed. Next we examined the effect of speed and gravity on the percent recovery of mechanical energy fluctuations of the center of mass in walking. Recovery is largely determined by $\alpha$ and the relative magnitudes of the fluctuations in $E_{p}$ and $E_{k h}$. We calculated the velocities at which $E_{p}$ and $E_{k h}$ fluctuated $180^{\circ}$ out of phase $(\alpha=$ $\left.0^{\circ}\right)$ and with equal magnitudes $\left(\mathrm{W}_{\mathrm{f}} \mathrm{W}_{\mathrm{v}}=1.0\right)$ at the four different levels of gravity (Fig. 3). Velocities were calculated for each subject and condition by applying a linear or second-order polynomial curve fit to the data and solving for the velocity according to the given criteria (e.g., $\alpha=0^{\circ}$ ). For nearly all the curve fits, $R>$ 0.9. At $1.00 \mathrm{G}, \alpha=0^{\circ}$ at $1.69 \pm 0.13(\mathrm{SE}) \mathrm{m} / \mathrm{s}$, and the magnitudes were equal at $1.23 \pm 0.14 \mathrm{~m} / \mathrm{s}$. Reducing gravity to $0.25 \mathrm{G}$ had a much greater effect on the velocity for $\alpha=0^{\circ}$ (75\% decrease to $0.42 \pm 0.23 \mathrm{~m} / \mathrm{s}$ ) compared with the velocity for equal magnitudes (21\% decrease to $0.97 \pm 0.10 \mathrm{~m} / \mathrm{s}$ ). Similarly, we calculated the velocity at which recovery was highest for each level of gravity (Fig. 3). At $1.00 \mathrm{G}$, maximum recovery occurred at $1.47 \pm 0.07 \mathrm{~m} / \mathrm{s}$. At $0.25 \mathrm{G}$, maximum recovery occurred at a slower vel ocity ( $40 \%$ decrease to $0.88 \pm 0.07 \mathrm{~m} / \mathrm{s}$ ).

Speed also had an effect on phase difference, relative magnitude, and recovery, as previously shown by 
Table 2. Data for walking at 1.00 and $0.50 \mathrm{G}$ at a range of vel ocities

\begin{tabular}{|c|c|c|c|c|c|c|c|}
\hline & $0.50 \mathrm{~m} / \mathrm{s}$ & $0.75 \mathrm{~m} / \mathrm{s}$ & $1.00 \mathrm{~m} / \mathrm{s}$ & $1.25 \mathrm{~m} / \mathrm{s}$ & $1.50 \mathrm{~m} / \mathrm{s}$ & $1.75 \mathrm{~m} / \mathrm{s}$ & $2.00 \mathrm{~m} / \mathrm{s}$ \\
\hline $\begin{array}{l}1.00 \mathrm{G} \\
0.50 \mathrm{G}\end{array}$ & $\begin{array}{l}38.4 \pm 4.2 \\
50.1 \pm 2.6\end{array}$ & $\begin{array}{l}54.9 \pm 4.2 \\
62.6 \pm 3.5\end{array}$ & $\begin{array}{l}64.0 \pm 2.9 \\
64.0 \pm 3.6\end{array}$ & $\begin{array}{l}65.4 \pm 2.9 \\
60.1 \pm 4.5\end{array}$ & $\begin{array}{l}66.1 \pm 2.0 \\
57.5 \pm 5.0\end{array}$ & $\begin{array}{l}64.7 \pm 2.3 \\
52.5 \pm 1.1\end{array}$ & $\begin{array}{l}59.9 \pm 4.1 \\
39.3 \pm 2.0\end{array}$ \\
\hline $1.00 \mathrm{G}$ & $0.60 \pm 0.10$ & $0.75 \pm 0.07$ & $0.92 \pm 0.09$ & $1.03 \pm 0.10$ & $1.16 \pm 0.12$ & $1.28 \pm 0.14$ & $1.48 \pm 0.18$ \\
\hline $0.50 \mathrm{G}$ & $0.84 \pm 0.10$ & $0.70 \pm 0.06$ & $0.93 \pm 0.05$ & $1.02 \pm 0.03$ & $1.18 \pm 0.04$ & $1.46 \pm 0.06$ & $1.91 \pm 0.01$ \\
\hline \multicolumn{8}{|l|}{$\alpha$, degrees } \\
\hline $1.00 \mathrm{G}$ & $0.14 \pm 0.01$ & $0.26 \pm 0.01$ & $0.40 \pm 0.02$ & $0.59 \pm 0.03$ & $0.81 \pm 0.04$ & $1.03 \pm 0.05$ & $1.23 \pm 0.08$ \\
\hline $0.50 \mathrm{G}$ & $0.08 \pm 0.01$ & $0.15 \pm 0.01$ & $0.22 \pm 0.02$ & $0.35 \pm 0.03$ & $0.45 \pm 0.04$ & $0.53 \pm 0.06$ & $0.66 \pm 0.01$ \\
\hline \multicolumn{8}{|c|}{$\mathrm{W}_{\mathrm{v}}, \mathrm{J} \cdot \mathrm{kg}^{-1} \cdot$ stride $^{-1}$} \\
\hline $1.00 \mathrm{G}$ & $0.26 \pm 0.04$ & $0.37 \pm 0.04$ & $0.47 \pm 0.06$ & $0.60 \pm 0.07$ & $0.73 \pm 0.08$ & $0.85 \pm 0.09$ & $0.90 \pm 0.11$ \\
\hline $0.50 \mathrm{G}$ & $0.10 \pm 0.01$ & $0.23 \pm 0.03$ & $0.25 \pm 0.02$ & $0.34 \pm 0.04$ & $0.38 \pm 0.04$ & $0.36 \pm 0.04$ & $0.35 \pm 0.01$ \\
\hline \multicolumn{8}{|c|}{$\mathrm{W}_{\text {ext }}, \mathrm{J} \cdot \mathrm{kg}^{-1} \cdot \mathrm{stride}^{-1}$} \\
\hline
\end{tabular}

Values are means $\pm S E ; n=6$ subjects except for the following vel ocities at $0.50 \mathrm{G}: 1.25 \mathrm{~m} / \mathrm{s}(\mathrm{n}=5), 1.50 \mathrm{~m} / \mathrm{s}(\mathrm{n}=4), 1.75 \mathrm{~m} / \mathrm{s}(\mathrm{n}=3)$, and 2.00 $\mathrm{m} / \mathrm{s}(\mathrm{n}=2)$. Subject number decreased at faster vel ocities at $0.50 \mathrm{G}$, because not all subjects were able to maintain a walking gait as defined by the position of the center of mass during single-limb support. See Table 1 footnote for definition of abbreviations.

Cavagna et al. (12) (Table 2). At velocities slower than the velocities where $\alpha=0^{\circ}$ and $\mathrm{W}_{\mathrm{f}} \mathrm{W}_{\mathrm{v}}=1.0, \alpha$ was positive (i.e., $E_{p}$ maximum preceded $E_{k h}$ minimum) and relative magnitudes $\left(\mathrm{W}_{\mathrm{f}} \mathrm{W}_{\mathrm{v}}\right)$ were $<1.0$. Conversely, at faster velocities, $\alpha$ was negative and relative magnitudes were $>1.0$. These general patterns applied to all the gravity conditions tested. The combined effects of speed and gravity on the percent recovery of mechanical energy are presented in Fig. 4. The spacing of the contour lines indicates the sensitivity of recovery to gravity and velocity. As noted by the broad plateau (i.e., broadly spaced contour lines), recovery remained relatively high at $>0.50 \mathrm{G}$ for walking velocities between 0.75 and $1.75 \mathrm{~m} / \mathrm{s}$. However, outside this range, recovery was much more sensitive to changes in speed or gravity (i.e., at $<0.50 \mathrm{G}$ and velocities of $<0.75$ or $>1.75$ $\mathrm{m} / \mathrm{s}$ ). This is clear from the closely spaced contour lines in Fig. 4. It is important to note that there is a slight

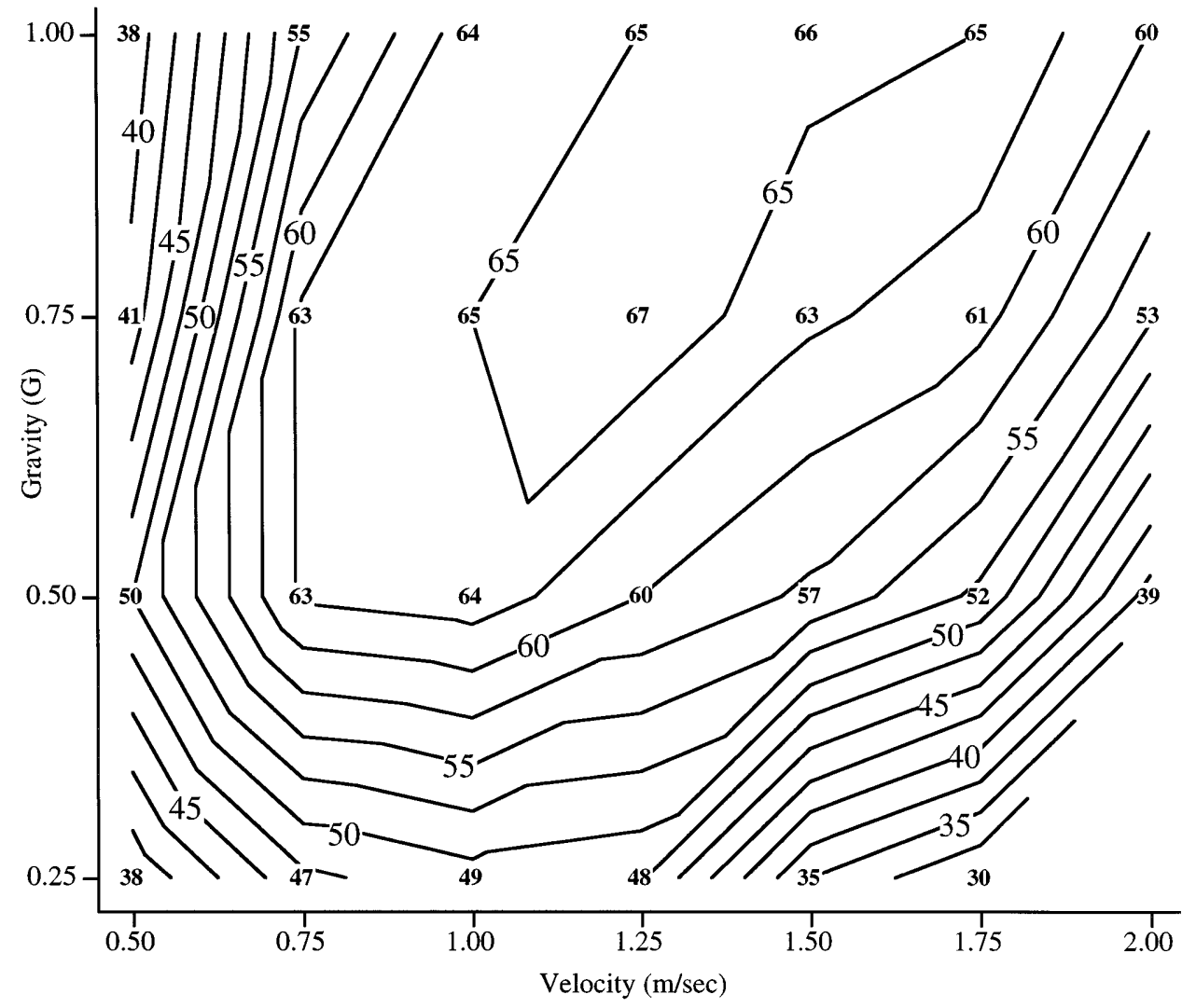

Fig. 4. Contour plot of percent recovery plotted against gravity and velocity. Each contour line indicates percent re covery of mechanical energy via inverted-pendulum mechanism. Small boldface numbers are mean values of experimental data for different combinations of gravity and velocity. Viewed like a topographical map, broadly spaced contour lines indicate a "plateau" region, where recovery remained high across large changes in gravity or vel ocity. Conversely, closely spaced contour lines indicate "cliff" regions, where recovery was sensitive to changes in gravity or velocity. Recovery remained high at $>0.50 \mathrm{G}$ and between 0.75 and $1.75 \mathrm{~m} / \mathrm{s}$. Contour lines were determined with DeltaGraph 4.0 (Deltapoint), which uses linear interpolations between empirical data points. 
difference between the calculated velocity values presented in Fig. 3 and the grouped mean values presented in Fig. 4. The reason for this small difference is that the vel ocity values in Fig. 3 were calculated from curve fits for each subject rather than grouped mean values.

Analysis of the position of the center of mass during the stance phase showed that all subjects were indeed walking at all gravity levels $(1.00-0.25 \mathrm{G})$ when vel ocities were $\leq 1.00 \mathrm{~m} / \mathrm{s}$. Consequently, all statistical tests were performed on data sets of equal subject number $(n=6)$. However, not all subjects were able to maintain a walking gait, as previously defined by the position of the center of mass, at faster velocities and lower levels of simulated gravity. As a result, trials that did not meet our criteria for walking were not included in any part of this study. In fact, at $0.25 \mathrm{G}$ and $2.00 \mathrm{~m} / \mathrm{s}$, the center of mass was closer to its lowest position at midstance in all the subjects. Thus no data were presented for the condition at $0.25 \mathrm{G}$ and $2.00 \mathrm{~m} / \mathrm{s}$ in Fig. 4, despite the fact that all subjects were walking according to a kinematic definition (i.e., maintaining at least 1 foot in contact with the ground throughout the entire stride).

\section{DISCUSSION}

Previous investigators have speculated that walking at a given moderate speed in reduced gravity would involve smaller fluctuations in $E_{p}$ but normal fluctuations in $E_{k h}(16,24,25)$. On the basis of this predicted mismatch of potential and kinetic energy, we made two hypotheses: 1) at a given moderate walking speed the recovery of mechanical energy would decrease as gravity is reduced, and 2) maximum recovery would occur at progressively slower speeds as gravity is reduced.

Overall, our data support our first hypothesis. At moder ate walking speeds (i.e., $1.00-1.50 \mathrm{~m} / \mathrm{s}$ ), recovery decreases as gravity is reduced (Fig. 4, Table 2). This effect is most pronounced at $1.50 \mathrm{~m} / \mathrm{s}$, whereas recovery is less affected by reducing gravity when walking at $1.00 \mathrm{~m} / \mathrm{s}$ (Table 1). Our data also support our second hypothesis. As gravity is reduced, maximum recovery occurs at progressively slower speeds (Fig. 3). This finding helps explain why recovery is only modestly affected by reduced gravity during walking at $1.00 \mathrm{~m} / \mathrm{s}$. However, it is important to note that our results do not agree with the underlying mechanism presumed by our hypotheses. Our hypotheses were based on a predicted mismatch of potential and kinetic energy in low gravity $(16,24,25)$. This prediction assumed that at a given speed the vertical displacements and forward velocity fluctuations of the center of mass would remain the same as gravity was reduced. Although we did find that the vertical displacements of the center of mass were unaffected by gravity (Table 1), the forward velocity fluctuations decreased as gravity was reduced. As a result, the fluctuations of potential and kinetic energy decreased proportionally in simulated reduced gravity. It appears that the effect of gravity on the phase difference between the fluctuations in potential and kinetic energy is the more important mechanism determining the effect of gravity on recovery.

One of our most intriguing findings was that the forward velocity fluctuations of the center of mass, as indicated by the $E_{k h}$ fluctuations, decreased at lower simulated gravity levels. This unpredicted decrease in forward velocity fluctuations could potentially be explained by a change in stride frequency. In normal gravity, $\mathrm{E}_{\mathrm{kh}}$ fluctuations may be increased or decreased by changing stride frequency at a given speed $(8,28)$. At stride frequencies greater than those freely chosen (i.e., shorter stride lengths), the fluctuations in $E_{p}, E_{k h}$, and $E_{\text {tot }}$ are reduced. Although data are not available for walking at $1.00 \mathrm{~m} / \mathrm{s}$, data from Cavagna and Franzetti (8) at $\sim 1.75 \mathrm{~m} / \mathrm{s}$ indicate that a $20 \%$ increase in step frequency results in an $\sim 50 \%$ decrease in $\mathrm{W}_{\mathrm{f}}$ and a $33 \%$ decrease in $W_{v}$. However, previous studies found that, in simulated reduced-gravity walking, stride frequency, as well as many other kinematic variables (e.g., ground contact time, duty factor, and leg swing time), undergo only small changes $(<10 \%)$ at moderate walking speeds $(14,16)$. In the present study, stride frequency at $1.00 \mathrm{~m} / \mathrm{s}$ did not exhibit any significant change at lower gravity levels $[0.84 \pm 0.02$ (SE) Hz, P = 0.29].

The primary mechanism underlying the decreased fluctuations in $\mathrm{E}_{\mathrm{kh}}$ appears to be simply changes in the horizontal braking and propulsive forces exerted on the ground. The peak horizontal force magnitudes and the horizontal impulses were reduced in near proportion to gravity. This proportional decrease of the vertical and horizontal components of the ground reaction forces allows the resultant ground reaction force vector to retain a similar orientation. This may be important for minimizing the muscle moments required at the joints. As Alexander (1) and many others have noted, during normal walking the resultant ground reaction force vector is aligned close to the hip joint throughout the stance phase.

Our findings also show that gravity and body size have similar effects on the inverted-pendulum mechanism in walking. The velocity at which $\mathrm{W}_{\mathrm{f}} \mathrm{W}_{\mathrm{v}}=1.0$ remained nearly unchanged by a fourfold change in gravity [1.10 \pm 0.05 (SE) m/s; Fig. 3]. Similarly, measurements comparing children with adults showed that the velocity at which $W_{f} / W_{v}=1.0$ was independent of size (velocity $1.25 \mathrm{~m} / \mathrm{s}$ ) (9). The phase difference values between the fluctuations of potential and kinetic energy are also affected in a similar manner by decreasing gravity or smaller size. At a given speed, lower gravity or smaller body size results in a negative shift in $\alpha$. Functionally, a negative phase shift indicates that the center of mass is still rising vertically as the horizontal propulsive phase begins. As a result, $\mathrm{E}_{\mathrm{p}}$ and $\mathrm{E}_{\mathrm{kh}}$ fluctuate $180^{\circ}$ out of phase at slower velocities as gravity is reduced or in smaller-sized individuals (Fig. 3) (9). Thus the velocity at which recovery is highest is dependent on gravity and body size.

Although we did not collect metabolic data, our findings may provide insight into the determinants of 


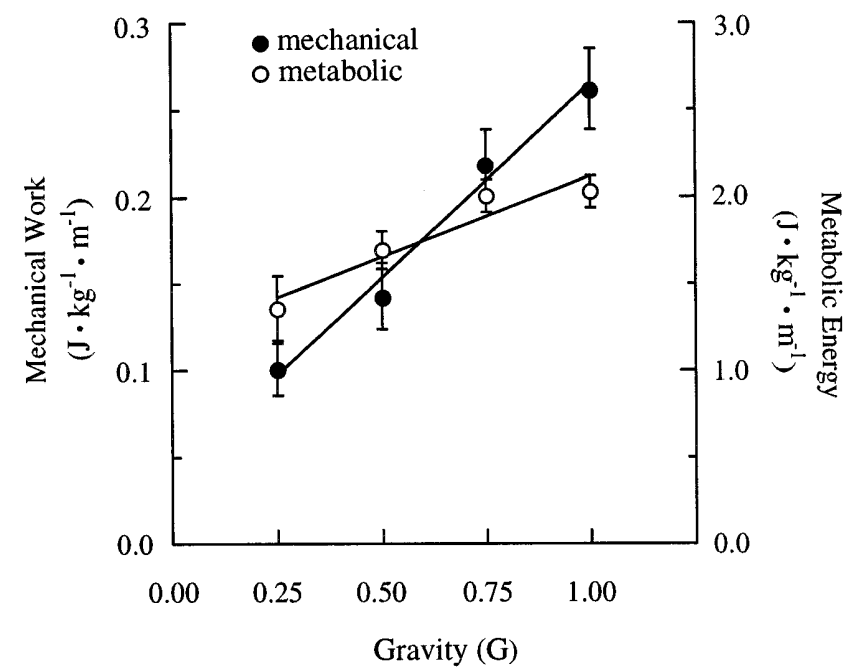

Fig. 5. External mechanical work per unit distance (left axis) de creased to a greater extent than metabolic cost of walking a unit distance (right axis) when gravity was reduced. Lines are leastsquares regressions: mechanical work $=0.04 \pm 0.22 \mathrm{G}, \mathrm{R}^{2}=0.671$, $95 \%$ confidence limits of slope $=0.13$ and $0.32, \mathrm{P}<0.01$; metabolic energy $=1.19 \pm 0.93 \mathrm{G}, \mathrm{R}^{2}=0.549,95 \%$ confidence limits of slope $=$ 0.45 and 1.41, and $P<0.01$. Data are for walking at $1 \mathrm{~m} / \mathrm{s}$. Mechanical values are means \pm SE for 6 subjects; metabolic values are means \pm SE for 4 subjects. Metabolic data are from Ref. 16.

the metabolic cost of walking. Recent studies indicate that both the cost of performing work and the cost of generating muscular forceto support body weight affect the metabolic cost of walking $(27,31)$. Farley and McMahon (16) found that when gravity is reduced by $50 \%$ the metabolic cost of walking decreases by only $25 \%$. Given that the amount of muscular force required to support body weight decreases in proportion to gravity, can the amount of work performed account for the less-than-proportional decrease in the metabolic cost of walking in simulated reduced gravity? We found that $\mathrm{W}_{\text {ext }}$ decreases by $45 \%$ at $0.50 \mathrm{G}$ (Fig. 5). Additionally, because our apparatus for simulating reduced gravity applied a force only about the center of mass, the work required to lift and accelerate the limbs relative to the center of mass was not affected and was presumably unchanged, since stride frequency did not change. Thus the amount of mechanical work performed does not appear to account for the relatively high (per unit weight) metabolic cost of walking in simulated reduced gravity.

Our results could beinterpreted in several ways. One interpretation might be that work is performed less efficiently in simulated reduced gravity. However, be cause subjects were allowed to choose their preferred walking mechanics, it seems likely that the subjects chose the most efficient movement pattern.

A second potential explanation for the relatively high metabolic cost of walking in reduced gravity is that the "effective mechanical advantage" (EMA) of the joint extensor muscles decreases in reduced gravity (4). EMA is the ratio of the muscle moment arm to ground reaction force moment arm. Consequently, if EMA decreased, larger muscle forces would be required to exert a given force on the ground, and the metabolic cost of generating force to support body weight would increase. However, kinematic data suggest that limb posture is similar in reduced-gravity walking. Donelan and Kram (14) found that stride length changed very little in simulated reduced gravity, and in the present study we found that the vertical displacements of the center of mass changed by $<10 \%$ over a fourfold change in gravity (Table 1). Also, as discussed earlier, the orientation of the ground reaction force vector about the joints does not appreciably change in reduced gravity, because $F_{z}$ and $F_{y}$ appear to decrease in proportion to gravity. Thus, although we did not rigorously examine the limb muscle's EMA, the present data suggest that it does not change in reduced gravity.

A third explanation that could account for the relatively high metabolic cost of walking in simulated reduced gravity is that the metabolic cost of swinging the limbs relative to the center of mass is more substantial than previously believed. When humans walk with a load in a backpack, the metabolic rate increases in proportion to the load while stride frequency does not change (23). These data, al ong with mechanical models and el ectromyogram evidence that leg swing is primarily passive at normal walking speeds $(3,29)$, suggest that the metabolic cost of swinging the limbs is small compared with the costs of performing external work and generating force to support body weight. However, recent walking studies indicate that leg swing may be more active than previously believed (14). Furthermore, if the center of mass and swing limb dynamics are coupled, as implied by Mochon and McMahon (29), the muscular work required to swing the limbs could be altered in our simulation of reduced gravity, because the center of mass accelerations decreases, despite a constant limb swing time. However, this proposed coupling between the center of mass and swing limb dynamics has yet to be resolved, inasmuch as Willems et al. (35) concluded that the most accurate measure of work in walking does not include energy transfer between the limbs and the center of mass of the whole body.

Finally, our findings may have implications for current rehabilitation programs used for spinal cordinjured patients. There is emerging evidencethat treadmill walking with partially supported body weight is helping some spinal cord-injured patients regain at least a limited ability to walk (33). Our results suggest that the fundamental mechanisms operating in normal walking also apply to partial body weight-supported walking given adequate spring compliance in the support mechanism. Walking in simulated reduced gravity is very similar to normal walking but with proportionately reduced forces.

\footnotetext{
The authors thank the University of California, Berkeley, Locomotion Laboratory for critical review and helpful comments in the preparation of the manuscript.

This research was supported by National Institutes of Health Grant R29-AR44688, the California Space Institute, the University of California President's UndergraduateF el lowship, the Undergraduate Research Apprentice Program, and the Biology Fellows Program.
} 
Address for reprint requests: T. M. Griffin, Dept. of Integrative Biology, 3060 Valley Life Sciences BIdg., University of California, Berkeley, CA 94720-3140 (E-mail: tmgriff@uclink4.berkel ey.edu).

Received 13 March 1998; accepted in final form 23 September 1998.

\section{REFERENCES}

1. Alexander, R. M. Energy-saving mechanisms in walking and running. J . Exp. Biol. 160: 55-69, 1991.

2. Alexander, R. M. Terrestrial locomotion. In: Mechanics and Energetics of Animal Locomotion, edited by R. M. Alexander and G. Goldspink. London: Chapman \& Hall, 1977, p. 168-203.

3. Basmajian, J. V., and C. De Luca. Muscles Alive: Their Function Revealed by Electromyography. Baltimore, MD: Williams \& Wilkins, 1985.

4. Biewener, A.A. Scaling body support in mammals: limb posture and muscle mechanics. Science 245: 45-48, 1989.

5. Blickhan, R., and R. J . Full. Locomotion energetics of ghost crab. II. Mechanics of the center of mass during walking and running. J . Exp. Biol. 130: 155-174, 1987.

6. Boda, W. L., D. E. Watenpaugh, R. E. Ballard, D. S. Chang, and A. R. Hargens. Comparison of gait mechanics and force generation during upright treadmill and supine LBNP exercise (Abstract). Med. Sci. Sports Exerc. 28: S87, 1996.

7. Cavagna, G. A. F orce platforms as ergometers. J . Appl. Physiol 39: 174-179, 1975

8. Cavagna, G. A., and P. F ranzetti. The determinants of the step frequency in walking in humans. J. Physiol. (Lond.) 373: 235$242,1986$.

9. Cavagna, G.A., P. Franzetti, and T. F uchimoto. The mechanics of walking in children. J . Physiol. (Lond.) 343: 323-339, 1983.

10. Cavagna, G. A., N. C. Heglund, and C. R. Taylor. Mechanical work in terrestrial locomotion: two basic mechanisms for minimizing energy expenditure. Am. J . Physiol. 233 (Regulatory I ntegrativeComp. Physiol. 2): R243-R261, 1977.

11. Cavagna, G. A., F. P. Saibene, and R. Margaria. External work in walking. J . Appl. Physiol. 18: 1-9, 1963.

12. Cavagna, G. A., H. Thys, and A. Zamboni. The sources of external work in level walking and running. J . Physiol. (Lond.) 262: 639-657, 1976.

13. Davis, B. L., and P. R. Cavanagh. Simulating reduced gravity: a review of biomechanical issues pertaining to human locomotion. Aviat. SpaceEnviron. Med. 64: 557-566, 1993.

14. Donelan, J . M., and R. Kram. The effect of reduced gravity on the kinematics of human walking: a test of the dynamic similarity hypothesis for locomotion. J . Exp. Biol . 200: 3193- 3201, 1997.

15. Farley, C. T., and T. C. Ko. Mechanics of locomotion in lizards. J . Exp. Biol. 200: 2177-2188, 1997.

16. Farley, C. T., and T. A. McMahon. Energetics of walking and running: insights from simulated reduced-gravity experiments. J . Appl. Physiol. 73: 2709-2712, 1992.
17. He, J . P., R. Kram, and T. A. McMahon. Mechanics of running under simulated low gravity. J . Appl. Physiol. 71: 863-870, 1991.

18. Hewes, D. E. Reduced-gravity simulators for studies of man's mobility in space and on the moon. Hum. Factors 11: 419-432, 1969.

19. Kram, R., A. Domingo, and D. P. Ferris. Effect of reduced gravity on the preferred walk-run transition speed. J . Exp. Biol. 200: 821-826, 1997

20. Kram, R., T. M. Griffin, J . M. Donelan, and Y. H. Chang. Force treadmill for measuring vertical and horizontal ground reaction forces. J . Appl. Physiol. 85: 764- 769, 1998.

21. Letko, W., A. A. Spady, and D. E. Hewes. Problems of man's adaptation to the lunar environment. In: Second Symposium on the Role of theVestibular Organs in SpaceExploration. Washington, DC: National Aeronautics and Space Administration, 1966, p. $25-32$.

22. Letko, W., and A.J . Spady. Walking in simulated lunar gravity. In: Fourth Symposium on the Role of the Vestibular Organs in Space Exploration. Washington, DC: National Aeronautics and SpaceAdministration, 1970, p. 347-351.

23. Maloiy, G. M., N. C. Heglund, L. M. Prager, G. A. Cavagna, and C. R. Taylor. E nergetic cost of carrying loads: haveAfrican women discovered an economic way? Nature 319: 668-669, 1986.

24. Margaria, R. Biomechanics and Energetics of Muscular Exercise Oxford, UK: Clarendon, 1976.

25. Margaria, R., and G. A. Cavagna. Human locomotion in subgravity. Aerospace Med. 35: 1140-1146, 1964.

26. McMahon, T. A., G. Valiant, and E. C. Frederick. Groucho running. J . Appl. Physiol. 62: 2326-2337, 1987.

27. Minetti, A. E., and R. M. Alexander. A theory of metabolic costs for bipedal gaits. J . Theor. Biol. 186: 467-476, 1997.

28. Minetti, A. E., C. Capelli, P. Zamparo, P. E. di Prampero, and F. Saibene. Effects of stride frequency on mechanical power and energy expenditure of walking. Med. Sci. Sports Exerc. 27: 1194- 1202, 1995.

29. Mochon, S., and T. A. McMahon. Ballistic walking. J. Biomech. 13: 49-57, 1980.

30. Newman, D. J ., H. L. Alexander, and B. W. Webbon. Energetics and mechanics for partial gravity locomotion. Aviat. Space Environ. Med. 65: 815- 823, 1994.

31. Taylor, C. R. Relating mechanics and energetics during exercise. Adv. Vet. Sci. Comp. Med. 38A: 181-215, 1994.

32. Thompson, D. A. W. On Growth and Form. Cambridge, UK: Cambridge University Press, 1942.

33. Wickelgren, I. Teaching the spinal cord to walk. Science 279: 319-321, 1998

34. Wickman, L. A., and B. Luna. Locomotion while load-carrying in reduced gravities. Aviat. Space Environ. Med. 67: 940-946, 1996.

35. Willems, P. A., G. A. Cavagna, and N. C. Heglund. External, internal and total work in human locomotion. J. Exp. Biol. 198: 379-393, 1995. 